\title{
Molecular diagnosis of gastric cancer: present and future
}

\author{
Wataru Yasui ${ }^{1}$, Naohide Oue ${ }^{1}$, Hiroki Kuniyasu ${ }^{1,2}$, Reiko Ito ${ }^{1}$, Eitchi Tahara ${ }^{3}$, and Hiroshi Yokozaki ${ }^{1}$ \\ ${ }^{1}$ First Department of Pathology, Hiroshima University School of Medicine, 1-2-3 Kasumi, Minami-ku, Hiroshima 734-8551, Japan \\ ${ }^{2}$ Department of Oncological Pathology, Cancer Center, Nara Medical University, Nara, Japan \\ ${ }^{3}$ Hiroshima Cancer Seminar Foundation, Hiroshima, Japan
}

\begin{abstract}
Although histopathological diagnosis is extremely useful for the definitive as well as the supportive diagnosis of gastric cancer in clinical practice, it is limited in certain respects. Over the past 15 years, integrated research in molecular pathology has clarified the details of genetic and epigenetic abnormalities of cancer-related genes in the course of the development and progression of gastric cancer. These abnormalities, which include telomerase activation, genetic instability, and abnormalities in oncogenes, tumor suppressor genes, cell-cycle regulators, cell adhesion molecules, and DNA repair genes, could be effective markers in the molecular diagnosis of gastric cancer. It is possible that the molecular analysis of these alterations in histopathology specimens may overcome deficiencies in diagnoses that depend only on histomorphology, and, consequently, we may be able to improve the differential diagnosis of cancer, obtain information on the grade of malignancy, and identify patients at high risk of developing multiple primary cancers. In Hiroshima, we have established a system of molecular-pathological diagnosis as a routine service; about 5000 lesions of the stomach have been subjected to this diagnosis, and much useful information has been obtained. In the near future, genetic analysis by means of DNA microarray may become routine in the diagnosis of gastric cancer. Genetic analysis of histopathology specimens may make clear the characteristics of individual cancers; indicating the common and specific features of molecular pathogenesis that may be directly connected with gene therapy or molecular-targeted therapy. By analyzing the relationship between singlenucleotide polymorphisms and cancer susceptibility, we will be able to obtain information on cancer prevention from histopathology samples.
\end{abstract}

Key words Molecular diagnosis - Gastric cancer · Histopathology $\cdot$ Genetic alterations $\cdot$ Epigenetic alterations

Offprint requests to: $\mathrm{W}$. Yasui

Received: May 21, 2001 / Accepted: July 3, 2001

\section{Introduction}

According to the Hiroshima Tumor Registration Committee, which registers tumors that have been diagnosed by pathologists, the number of cases registered has increased threefold for gastric cancer and 20 -fold for gastric adenoma over the past 20 years. This suggests that, recently, many tumors have been biopsied or dissected endoscopically, while tumor tissues were collected only by surgery in earlier periods. Although histopathological diagnosis is, thus, extremely important for obtaining definitive and supportive diagnoses, diagnosis that depends only on histopathology has certain limitations. Many lesions have a morphology which is between that of benign and malignant lesions, and differential diagnosis is therefore difficult. Variations between pathologists may lead to changes in assessments of the lesion. Differences in diagnostic criteria, such as those for early gastrointestinal cancer diagnosed by Western and Japanese pathologists, lead to misunderstanding by clinicians. Furthermore, information derived from morphology is of limited use in determining the degree of malignancy and the patient's prognosis. No genetic information can be obtained in hereditary cancers, such as hereditary non-polyposis colorectal cancer (HNPCC) kindreds.

Integrated research in molecular pathology over the past 15 years has clarified details of the genetic and epigenetic abnormalities of cancer-related genes in the course of the development and progression of gastric cancer [1-3]. Because most of these alterations can be analyzed in the formalin-fixed specimens that are taken for the purpose of histopathological diagnosis, these alternations are good targets for molecular diagnosis [4-7]. It is possible to apply molecular findings in tissue samples to diagnosis, together with the results of morphology. Thus, molecular diagnosis can overcome the drawbacks of histopathological diagnosis and can make 
a great contribution to the understanding of cancer histopathology.

This review outlines the procedure for molecular diagnosis in gastric specimens that has been routinely implemented in Hiroshima. An overview of the genetic and epigenetic alterations in gastric cancer is given, to show the significance of these changes as biological markers in diagnosis. We also discuss future prospects for the molecular diagnosis of gastric cancer.

\section{Molecular basis of multistep stomach carcinogenesis}

In the course of multistep carcinogenesis in the stomach, various genetic alterations of oncogenes, tumor suppressor genes, DNA repair genes, cell-cycle regulators, and cell adhesion molecules are accumulated (Fig. 1) [1-3]. Of the various genetic and epigenetic alterations, some are found in both well and poorly differentiated types of carcinoma, while others are found in only one particular histological type. Genetic instability, inactivation of tumor suppressor genes by $\mathrm{CpG}$ island methylation, and telomerase activation commonly participate in the initial step of stomach carcinogenesis. While p53 gene loss and mutation occurs at an early stage of carcinogenesis, amplification of the c-met and cyclin $\mathrm{E}$ genes is frequently associated with an advanced stage. Reduced expression of p27 ${ }^{\mathrm{Kip} 1}$, a cyclin-dependent kinase (CDK) inhibitor, participates in both the development and the progression of gastric cancer. Overexpression of growth factors and cytokines partipates in progression. K-ras mutations, amplifica- tion of the c-erbB2 gene, inactivation of the $A P C$ gene, and loss of $\mathrm{pS} 2$ expression preferentially occur in the well differentiated type. Precancerous lesions, such as intestinal metaplasia and adenoma, share various alterations similar to those seen in well differentiated carcinomas. Loss of heterozygosity (LOH) of the $p 73$ gene occurs specifically in well differentiated carcinomas with foveolar epithelial phenotype [8]. Inactivation of cadherins and amplification of the K-sam gene and c-met gene are frequently associated with poorly differentiated or scirrhous type carcinomas. A review of representative abnormalities of individual genes and molecules, and their significance as markers for molecular diagnosis, is given below.

Telomere maintenance by telomerase activation induces cellular immortalization [9]. Strong telomerase activity is detected in most gastric carcinomas, regardless of histological type and tumor stage [10]. Relatively good correlation was found between telomerase activities and levels of catalytic subunit of telomerase (TERT) expression in gastric carcinomas. Some intestinal metaplasias and gastric adenomas express telomerase activity at certain levels, suggesting participation in an early stage of stomach carcinogenesis [11]. Telomerase activity is found in approximately half of gastric adenomas, whose levels of the activity are about $10 \%$ of those in gastric carcinomas. TERT protein is expressed in the nuclei of the adenoma cells at moderate levels that are not necessarily comparable with the telomerase activity. Therefore, TERT expression may be a prerequisite for telomerase activation in the early stage of stomach carcinogenesis; thus, the expression of

\section{Well differentiated adenocarcinoma}

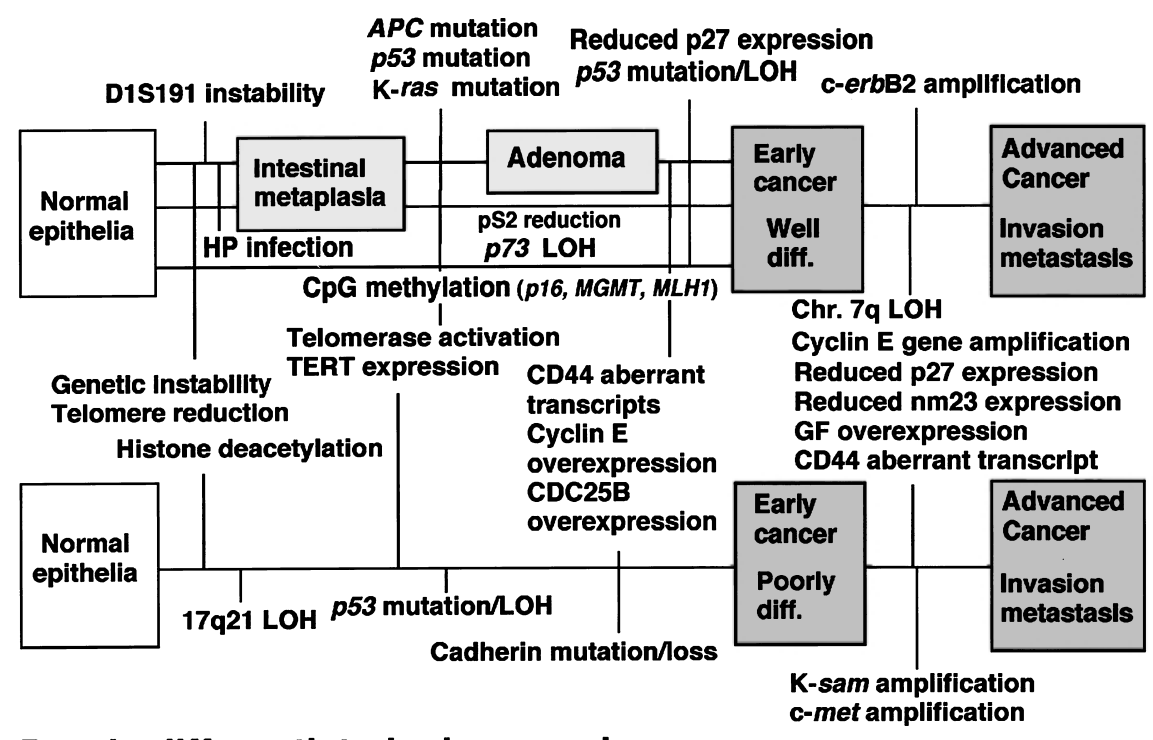

Poorly differentiated adenocarcinoma
Fig. 1. Genetic and epigenetic alterations during multistep stomach carcinogenesis. $\mathrm{LOH}$, Loss of heterozygosity; HP, Helicobacter pylori; TERT, catalytic subunit of telomerase; Chr., chromosome; $G F$, growth factors 
TERT is a useful marker for detecting "true precancerous lesions".

Genetic instability causes an accumulation of genetic alterations, and participates in the early stage of stomach carcinogenesis [3]. Cases in which two or more of five microsatellite loci show replication error are considered as showing high-frequency microsatellite instability (MSI-H), and those with only one locus showing replication error are considered to show low-frequency MSI (MSI-L) [12]. MSI-H is observed in only $4 \%$ of gastric carcinomas, in particular in the well differentiated type in aged patients. The frequency of MSI-L is about $30 \%$ in primary gastric cancers, including early cancers [3]. Some intestinal metaplasias and adenomas also show MSI-L, and these should be considered "true precancerous lesions" [13]. MSI-H is used as an indicator of HNPCC, which is caused by the germline mutation of mismatch repair genes, including $h M L H 1$ and $h M S H 2$ [14]. In sporadic gastric cancers with MSI-H, $\mathrm{CpG}$ island hypermethylation of $h M L H 1$ is associated with loss of hMLH1 protein $[15,16]$. Another important aspect of MSI is that multiple primary cancers, including stomach, colon, and gallbladder carcinomas, arising in a single individual frequently display replication errors at multiple microsatellite loci [17]. This indicates that the detection of MSI in a cancer may serve as a good molecular marker for the assessment of the risk of a second cancer in the same patient.

Abnormalities in cell-cycle regulators are involved in the development and progression of gastric cancers through unbridled cell proliferation $[2,3]$. The cyclin E gene is amplified in $15 \%-20 \%$ of gastric carcinomas. The overexpression of cyclin $\mathrm{E}$ tends to be correlated with the aggressiveness of gastric carcinomas, such as deep invasion and advanced tumor stage. On the other hand, reduction in the expression of the CDK inhibitor, p27 ${ }^{\mathrm{Kip} 1}$, is frequently associated with advanced gastric carcinomas [18]. Gastric adenomas with reduced p27Kip1 expression tend to progress to carcinomas [7]. Reduced expression of $\mathrm{p} 27^{\mathrm{Kip} 1}$ also significantly correlates with depth of tumor invasion and the presence of lymphnode metastasis. Therefore, gene amplification and the overexpression of cyclin E, and the reduced expression of p27 ${ }^{\mathrm{Kip} 1}$, are good markers for the diagnosis of highgrade malignancy. E2F-1, a target of cyclins/CDKs at G1/S transition, is overexpressed in about $40 \%$ of gastric carcinomas and may participate in the development of gastric carcinomas [19].

Abnormalities in transcriptional regulation by $\mathrm{CpG}$ island methylation and histone deacetylation are believed to be the most important of various epigenetic alterations [20-23]. DNA hypermethylation is known to be accompanied by allelic loss at the same locus. CpG island hypermethylation of the genes $h M L H 1$, $M G M T\left(\mathrm{O}^{6}\right.$-methylguanine methyltransferase $)$, and p16 ${ }^{\text {MTSIIINK4A }}$ is detected in $20 \%-30 \%$ of gastric cancers [24-26]. Reduced expression of the gene products is well correlated with $\mathrm{CpG}$ island hypermethylation. Hypermethylation of these three genes is more frequently found in well differentiated adenocarcinomas than in poorly differentiated adenocarcinomas. However, cases with $h M L H 1$ hypermethylation do not coincide with cases with $M G M T$ hypermethylation, as reported in colorectal cancer. Furthermore, hMLH1 hypermethylation occurs in intestinal metaplasia, suggesting that hMLH1 expression reduced by promoter methylation may be an initial event in the accumulation of genetic abnormalities in stomach carcinogenesis. On the other hand, $\mathrm{CpG}$ island methylation of CDH-1 (E-cadherin) and consistently reduced E-cadherin expression is commonly observed in scirrhous gastric cancers. Although the enzymes DNA methyltransferase and demethylase potentially affect promoter methylation status, the tumor-specific hypermethylation of $h M L H 1, p 16^{M T S 1 / I N K 4 A}$, and $C D H-1$ does not simply depend on the expression levels of promethylating (DNMT1, DNMT3A, DNMT3B) and antimethylating (MBD2) enzymes $[24,26]$.

Nucleosomes consist of core histones, wrapped with turns of double-stranded DNA [20]. The acetylation of histones disrupts nucleosome structure, leading to DNA relaxation, and a subsequent increase in accessibility for transcription factors. On Western blotting, with anti-acetylated histone $\mathrm{H} 4$, the level of acetylated histone $\mathrm{H} 4$ expression was significantly reduced in $70 \%$ of gastric carcinomas in comparison with findings in normal mucosa, indicating a reduced acetylation status in gastric cancers. The histone deacetylase inhibitor, trichostatin A (TSA), inhibits cell growth and induces apoptosis in gastric carcinoma cell lines [27]. TSA induces the expression of $\mathrm{p} 21^{\mathrm{WAF} 1 / \mathrm{cip} 1}, \mathrm{CBP}, \mathrm{Bak}$, and cyclin E, while it reduces the expression of E2F-1, E2F4, HDAC-1, and the phosphorylated form of Rb protein [27]. These findings suggest that histone deacetylation may participate in unbridled cell proliferation by modulating the expression of cell-cycle regulators and apoptosis-related molecules. The expression of acetylated histone $\mathrm{H} 4$ is also reduced in $40 \%$ of gastric adenomas and some intestinal metaplasias adjacent to carcinoma, suggesting that reduced histone acetylation may occur even in precancerous cells, and may be a candidate molecular marker to indicate such cells.

\section{Molecular diagnosis of gastric cancer on histopathology specimens}

Based on the molecular pathological findings mentioned above, it is clear that we can improve the quality of histopathological diagnosis by analyzing genetic and 
epigenetic alterations. We established a molecular diagnosis system for histopathological samples of gastrointestinal tract, in cooperation with Hiroshima City Medical Association Clinical Laboratory, and we have been performing this as a routine service [6,7]. This system is designed mainly for the differential diagnosis of benignancy or malignancy, diagnosis of the degree of malignancy, identification of susceptibility to multiple primary cancers, and identification of HNPCC.

\section{Procedures for molecular diagnosis}

The procedures used, from sample receipt to the final diagnosis in the pathology laboratory, are outlined in Fig. 2. The samples are formalin-fixed tissue materials obtained by biopsy or dissection (endoscopic mucosal resection or surgical removal) that are submitted for routine histopathological diagnosis. Routine paraffin sections are prepared, stained, and subjected to histopathological observation. A pathologist indicates the cases to be examined for molecular diagnosis (cancer, adenoma [dysplasia] or borderline lesion) at microscopic examination. A laboratory technician performs immunostaining with various molecular and genetic markers, described below, and at that time prepares another five sections with $\mathrm{H} \& \mathrm{E}$ staining that are not included by covering glass for genetic analysis. The pathologist combines the results of immunostaining and the histopathological findings, adds the molecular pathological findings and their significance if new information is obtained, and makes a molecular-pathological report for the attending clinicians.

Some cases are further analyzed using DNA extracted from paraffin sections. In order to assure that only the histologically doubtful portion is examined accurately, the position to be analyzed on the section with
H\&E staining is marked with a felt pen under the microscope by the pathologist. A technician collects the tissues on the marked area into an Eppendorf tube, using the tip of the needle, and extracts DNA using a conventional method with proteinase $\mathrm{K}$ and sodium dodecylsulfate (SDS). For this extracted DNA, mutation or deletion of the genes of interest is examined by nonisotopic polymerase chain reaction single-strand conformation polymorphism (PCR-SSCP) analysis and PCR restriction fragment length polymorphism (RFLP). For the detection of genetic instabilities, microsatellite loci are amplified with PCR, using fluorescent stain-labeled primers, and analyzed with a PRISM 310 autosequencer (Applied Biosystems, Foster City, CA, USA). The final molecular-pathological diagnosis, made by combining the results of genetic analysis, immunostaining, and histopathological findings, is reported to the clinicians.

The immunostaining, DNA extraction, PCR-SSCP, and PCR-RFLP are conducted by technicians in the clinical laboratory center of Hiroshima City Medical Association, and microsatellite analysis is performed at our department (First Department of Pathology, Hiroshima University School of Medicine). Most expenses are covered by a donation from the Hiroshima City Medical Association and by research funds from our department. The molecular pathologists who are in charge of the molecular pathological diagnosis system work voluntarily.

\section{Molecular and genetic markers}

As the significance of each genetic and epigenetic abnormality differs in esophageal, gastric, and colorectal cancers, the roles of individual abnormalities should be sufficiently understood before these abnormalities are used in diagnosis. In gastric cancer, p53, APC, and

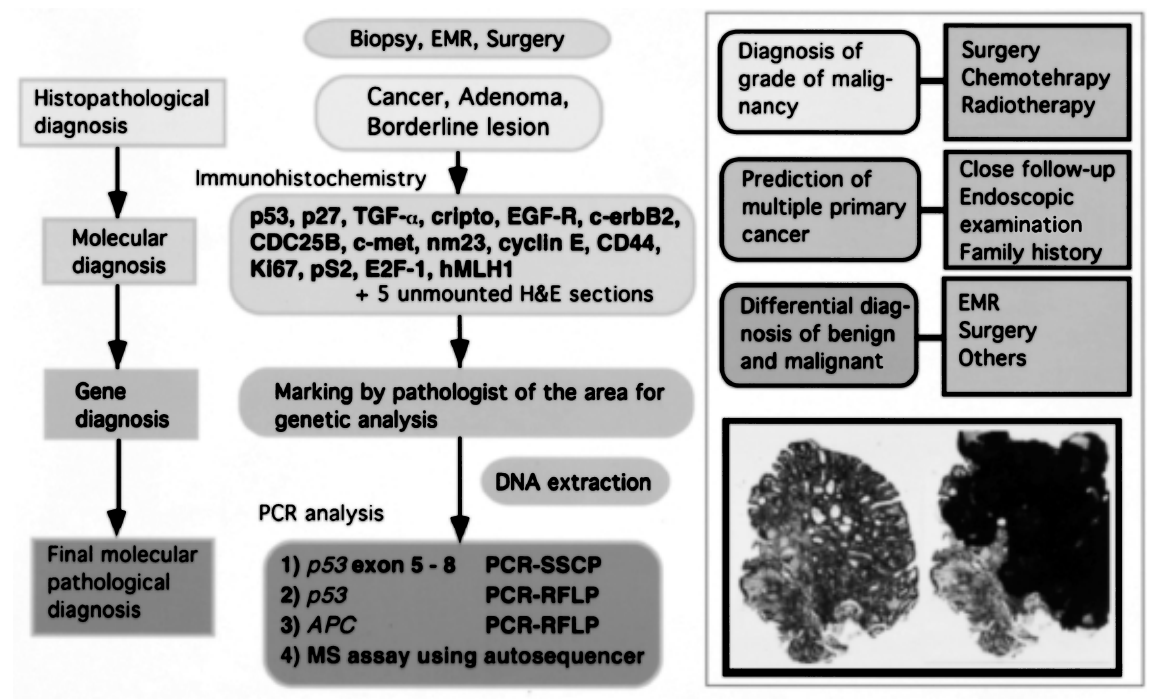

Fig. 2. Procedures for the molecularpathological diagnosis of histopathology samples of the stomach. EMR, Endoscopic mucosal resection; $T G F-\alpha$, transforming growth factor alpha; EGFR, epidermal growth factor receptor; $P C R$, polymerase chain reaction; $S S C P$, singlestrand conformation polymorphism; $R F L P$, restriction fragment length polymorphism; $M S$, microsatellite 


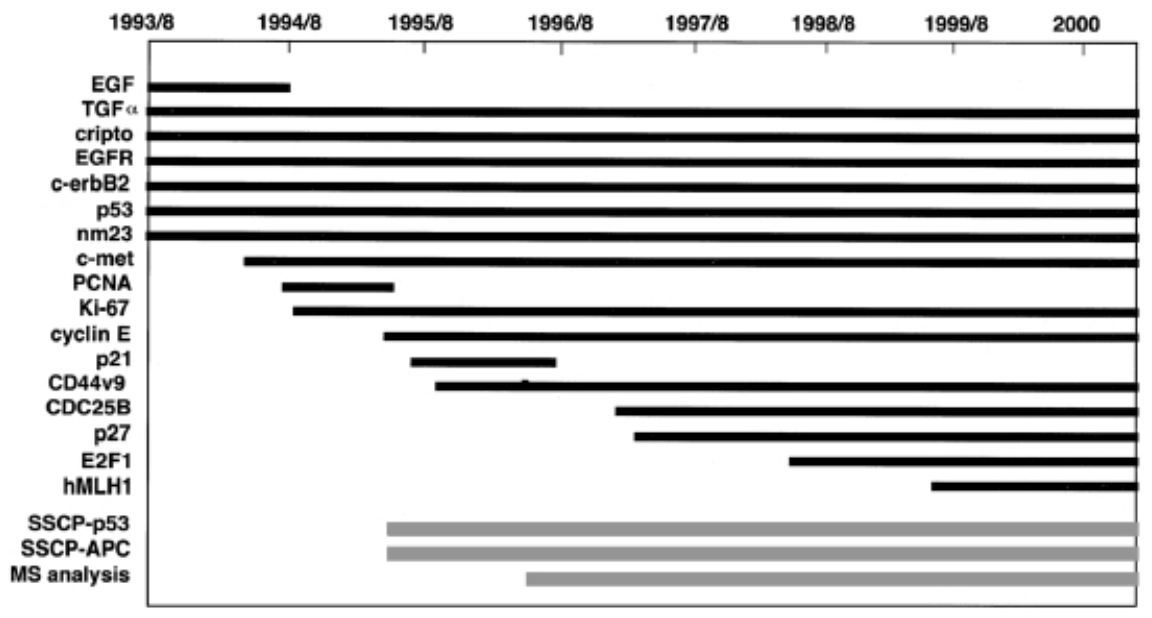

Fig. 3. Changes in molecular and genetic markers for molecular-pathological diagnosis of the stomach during the 7 years that our system has been operating. $P C N A$, Proliferating cell nuclear antigen

Table 1. Molecular-pathological diagnosis of gastric lesions

\begin{tabular}{lclc}
\hline Histological diagnosis & Lesions & \multicolumn{2}{c}{ Molecular diagnosis } \\
\hline Adenoma & \multirow{2}{*}{1154} & $\begin{array}{l}\text { Adenoma with malignant potential } \\
\text { s/o Adenocarcinoma }\end{array}$ & $119(10 \%)$ \\
Borderline & \multirow{2}{*}{469} & $\begin{array}{l}\text { Adenoma with malignant potential } \\
\text { s/o Adenocarcinoma }\end{array}$ & $6(1 \%)$ \\
& & Adenocarcinoma & $45(10 \%)$ \\
s/o Adenocarcinoma & \multirow{2}{*}{250} & Adenocarcinoma & $53(11 \%)$ \\
Adenocarcinoma & \multirow{2}{*}{2969} & High grade malignancy & $63(25 \%)$ \\
& & High-grade malignancy & $3(1 \%)$ \\
& & & $357(12 \%)$ \\
\hline
\end{tabular}

s/o, Suspected of

CD44 have been used as markers for differential diagnosis, and transforming growth factor alpha (TGF $\alpha$ ), epidermal growth factor receptor (EGFR), c-met, cerbB2, cyclin E, p27 ${ }^{\mathrm{Kip} 1}$, and CDC25B for degree of malignancy. For the screening of genetic instability, hMLH1 expression is examined. With PCR-SSCP and PCR-RFLP, deletions or mutations of the $A P C$ gene and the $p 53$ gene are examined. Mutations of the $p 53$ gene are analyzed in exons 5 to 8 , and $\mathrm{LOH}$ is analyzed using polymorphism at the BamH1 site of the 3'untranslated region of the gene. Genetic instability is monitored by microsatellite assay. Four loci of two CA repeats (D1S191, D17S855; BRCA1 locus) and two poly A tracts (BATRII; TGF $\beta$ type II receptor and BAT40) are examined, and if two or more loci with replication errors are detected, MSI-H is considered to be present. For MSI-H, the presence or absence of mutation of $h M L H 1$ and $h M S H 2$ is determined by PCR-SSCP methods.

The assessment of biomarkers is reviewed periodically, and we always study new markers retrospectively to verify their usefulness for diagnosis prior to use in clinical practice. Figure 3 shows the history of molecular markers for stomach specimens during the 7 years that our system has been operating. In 1993, molecular diag- nosis was begun with immunostaining alone, with only 6 markers; genetic analysis of the $p 53$ and $A P C$ genes by PCR-SSCP and PCR-RFLP was introduced in 1995; and, since 1996, microsatellite analysis has been performed with an autosequencer. At present, we analyze 14 molecular and 3 genetic markers.

\section{Results and evaluation}

Approximately 5000 lesions in histopathological samples of the stomach have been analyzed. The histopathological diagnoses are adenoma, dysplasia, borderline lesion, carcinoma, and lesion suspected of carcinoma. The results of molecular-pathological diagnosis of gastric lesions are summarized in Table 1. As mentioned above, molecular diagnosis is made by combining the results of molecular and genetic analyses and the histopathological findings. The definitions we use in molecular diagnosis are as follows: (1) "adenoma with malignant potential" is an adenoma with a high probability of becoming carcinoma. These tumors contain some molecular and genetic abnormalities, including $p 53$, but morphological aberrations are within the range for benign tumor. (2) "Suspected of ( $/ \mathrm{o}$ ) adenocarcinoma" is a tumor that has genetic abnormalities of $p 53$ 


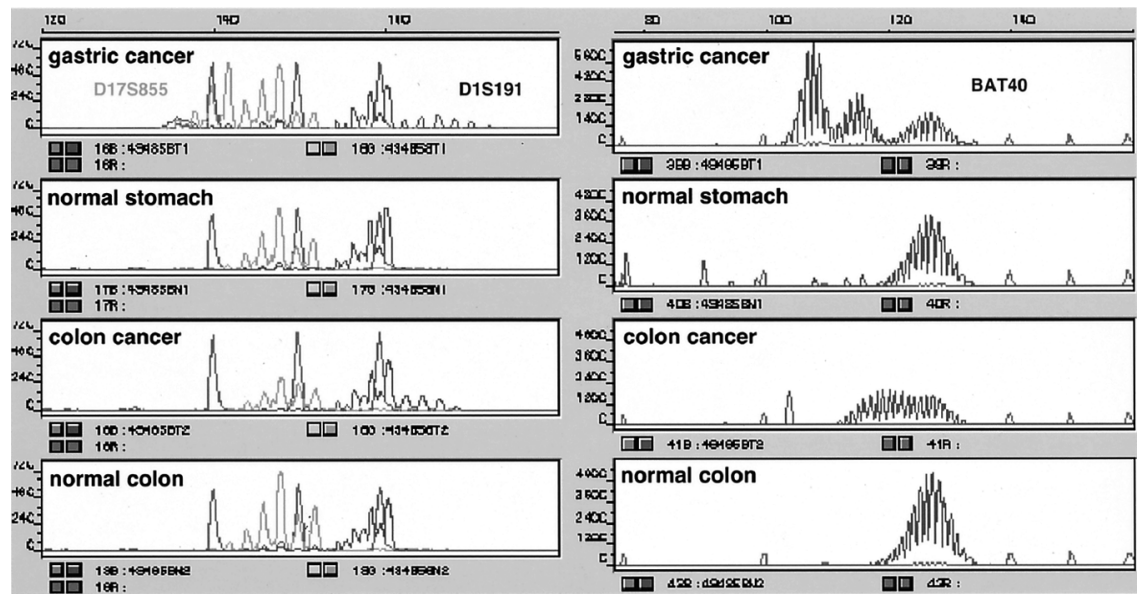

Fig. 4. A representative case of double cancer (gastric and colon cancers) with high-frequency microsatellite instability (MSI-H). DNA was extracted and subjected to microsatellite assay, as described in the text. Three loci (D17S855, D1S191, and BAT-40) were shown in both the gastric and the colon cancers, but not in the normal counterparts and/or $A P C$, as well as abnormal expression of many cancer-related molecules, such as c-erbB2, cyclin E, EGFR, and c-met, while histological atypia is not severe enough to be regarded as carcinoma. (3) "Adenocarcinoma" is a tumor whose genetic and molecular abnormalities are the same degree as those in (2) above, that can be also regarded as malignant histopathologically if such molecular findings are available. Ten percent of histologically diagnosed adenomas were diagnosed as adenoma with malignant potential, while $2 \%$ were suspected of adenocarcinoma. Adenocarcinoma or s/o adenocarcinoma was identified in more than $20 \%$ of histologically diagnosed borderline lesions. Twelve percent of histologically diagnosed adenocarcinomas were regarded as showing high-grade malignancy because of abnormalities of growth factors or growth factor receptors, cell-cycle regulators, and so on, such as c-erbB2, EGFR, cyclin E, and p27 Kip1. The prognosis of patients diagnosed with these abnormalities tended to be poor on follow-up observation. About $4 \%$ of gastric cancers exhibited MSI-H by microsatellite analysis (Fig. 4). In half of the patients who showed MSI-H, clinically synchronous or asynchronous multiple primary cancers were confirmed. We found germline as well as somatic mutations of DNA mismatch repair genes in some cases, suggesting a kindred of HNPCC, but most cases showed epigenetic inactivation of $h M L H 1$, possibly caused by $\mathrm{CpG}$ island methylation.

\section{Points at issue}

With our diagnosis system, the portion of the section used for DNA extraction is marked with a felt pen, and tissue in the marked area is collected as accurately as possible. One of the technical problems with this system is that accurate sample collection is sometimes difficult, and genetic analysis cannot be done in cases in which a small number of cancer cells are distributed in the nor- mal epithelia, such as in signet ring cell carcinoma, or when cancer cells are infiltrated diffusely in fibrous stroma, such as in scirrhous carcinoma. To deal with these problems, we are introducing laser microdissection [28] for routine use. With this technique, tumor cells can be distinctly separated from normal mucosal epithelia.

Another problem with our system is that we do not have enough molecular markers; we need additional markers, e.g., for differential diagnosis, grade of malignancy, and drug sensitivity, to make diagnosis more efficient. The DNA microarray technique is a powerful tool to identify novel genes participating in the development and progression of gastric cancer. Figure 5 shows the representative result of a comparative gene expression profile of well differentiated adenocarcinoma and poorly differentiated scirrhous gastric carcinoma obtained using the DNA microarray technique. Strong expression of cadherin, cell-cycle regulators, and mitogen-activated protein (MAP) kinases was observed in the well differentiated type, while survivin, growth factors (TGF $\beta$, platelet-derived growth factor [PDGF], insulin-like growth factor [IGF]), and proteases (matrix metalloproteinase [MMP]-2, MMP-3, plasminogen activator $[\mathrm{PA}]$ ) were found to be strongly expressed in scirrhous type carcinoma. Large numbers of genes are differentially expressed in various tumors, but their role in stomach carcinogenesis is not known. Further studies should be performed to verify the value of such genes in molecular diagnosis, both retrospectively and prospectively.

\section{Another contribution of molecular analysis to clinicical findings}

Super-early diagnosis of gastric cancer

As described above, many genetic and epigenetic abnormalities are found in incomplete type intestinal 

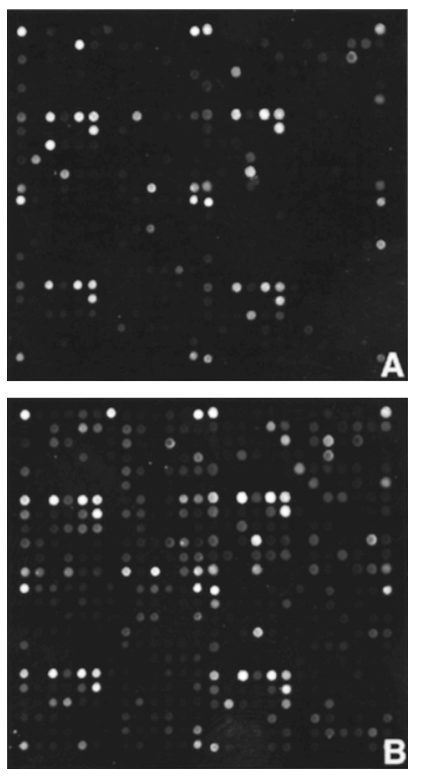

Fig. 5.A,B. Differential gene expression of well differentiated adenocarcinoma (A), detected by $\mathrm{Cy} 5$, and poorly differentiated scirrhous carcinoma (B) detected by $\mathrm{Cy} 3$, using cDNA microarray

metaplasia without obvious morphological aberration. Therefore, if molecular analysis is extended to intestinal metaplasia, super-early diagnosis of gastric cancer can be made. For instance, mutations of the $p 53, A P C$, or Kras genes have been detected in intestinal metaplasia, although at low frequency [29]. The intestinal metaplasia in $30 \%$ of gastric cancer cases shows weak but significant telomerase activity, suggesting the existence of "stem-cell hyperplasia" or "already immortalized somatic cells" in intestinal metaplasia [3]. TERT protein is expressed in some incomplete-type intestinal metaplasias adjacent to carcinomas [11]. The expression of acetylated histone is reduced in intestinal metaplasia. Examinations of foci with genetic instability in stomach resected because of gastric cancer have shown, replication error-positive foci located both in the cancer and in the intestinal metaplasia around the cancer [13]. Some intestinal metaplasias show $\mathrm{CpG}$ island hypermethylation of the $h M L H 1$ gene and its reduced expression (Fig. 6). Foci with replication error and portions with reduced $h M L H 1$ expression in nonneoplastic mucosa are lesions that are susceptible to cancer progression (true precancerous lesions), and genetic instability is a powerful genetic marker to predict such a condition.

\section{Diagnosis of presence of cancer}

It is needless to state that lymph node metastasis is the most important determinant of patient outcome. However, with routine histopathological examination of representative sections of the cut surface of lymph nodes, there is a chance that micrometastasis may be overlooked. The molecular detection of mRNAs expressed in cancer cells but not in other cells in lymph nodes,

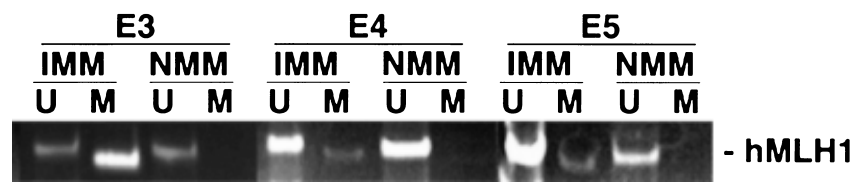

Fig. 6. $\mathrm{CpG}$ island hypermethylation of the $h M L H 1$ gene in intestinal metaplasia. The methylation status of the $\mathrm{CpG}$ island in the promoter of the $h M L H 1$ gene was examined in intestinal metaplastic mucosa $(I M M)$ and non-metaplastic mucosa $(N M M)$ by a methylation-specific PCR method

such as the mRNA for keratin 19 or carcinoembryonic antigen (CEA) is useful for detecting micrometastasis [30,31]. The expression of these genes can be found by reverse transcriptase (RT)-PCR in many lymph nodes that are histologically negative for metastasis. Histologically node-negative patients with micrometastasis detected by means of cytokeratin expression show worse prognosis than those without micrometastasis [32].

The detection of cancer cells in the peritoneal cavity has important prognostic implications. Routine cytological examinations, however, are not sensitive, and gastric cancer patients with negative peritoneal cytology sometimes show peritoneal recurrence. The analysis of CEA mRNA expression by RT-PCR is sensitive enough to detect free cancer cells in peritoneal washes that are negative for abnormal cytology, indicating that such analysis is a powerful technique for predicting the risk of peritoneal dissemination. Real-time RT-PCR is more rapid and quantitative than the conventional one, and is applicable to decision-making during surgery [33]. A similar strategy can be employed for diagnosing the presence of cancer cells in peripheral blood [34]. $C E A$ mRNA from circulating cancer cells is associated with an advanced disease stage, and is a useful diagnostic modality to determine patient selection for chemotherapy after surgery and to determine the patient's prognosis.

\section{Future prospects for molecular diagnosis}

The human genome sequence, determined by both the International Human Genome Sequencing Consortium and Celera Genomics of Rockville, was published in Nature of 15 February 2001 [35], and in Science of 16 February 2001 [36], respectively. Although the project is not yet completed, approximately 3 billion base pairs were sequenced, covering $90 \%$ of the euchromatic portion of the human genome. The number of proteincoding genes is estimated to be about 30000 . We believe that this determination of the human genome sequence is the greatest scientific achievement made in the past decade. Because one principle of the Human Genome 
Project, reflected in the Universal Declaration of the Human Genome and Human Rights, is unlimited access to the sequence, the entire information on the human genome in Nature is available without restriction (http://www.nature.com.genomics). This is the beginning of the post-sequence era. DNA microarray technology has been established, and with this technology, information on the mutation and expression of many genes in human cancers can be quickly detected, stored, and analyzed. It is a time of evolution in that molecular diagnosis in the field of pathology must correspond to this new scientific age. The findings that have already accumulated on the molecular mechanisms of the development and progression of gastric cancer, and findings that will be systematically clarified in the future will be combined, and genetic diagnosis by means of DNA microarray will surely become routine in the diagnosis of gastric cancer. If essential abnormalities and secondary changes can be clarified in the many genetic and epigenetic alterations identified in cancers, examinations of a hundred to several hundred genes should be sufficient for the diagnosis of gastric cancer. It is important that the characteristics of each cancer, that is, the common features and specific features in the development and progression of each cancer, are clarified so that gene therapy or molecular-targeted therapy can be directly related to the results of molecular diagnosis of histopathological samples. We plan to create DNA miniarrays for the diagnosis of gastric cancer to predict the patient's susceptibility and the grade of malignancy and chemosensitivity of the cancer.

Most genetic variation between individual humans is believed to be caused by single-nucleotide polymorphisms (SNPs). The information on SNPs identified to date is publicly available. Although the average frequency of SNPs is estimated to be 1 bp per 1.2 to $1.9 \mathrm{kbp}$, fewer than $1 \%$ of all SNPs result in variations in proteins [37]. Although the tasks of determining which SNPs have a functional consequence remain, largely, to be elucidated, some evidence has been found in relation to pathological status. Genetic polymorphism of the $C Y P$ genes is related to cancer predisposition, including lung cancer. SNP in the RAD51 gene modifies breast cancer risk in $B R C A 2$ carriers [38]. SNP in the interleukin-1- $\beta$ gene is associated with an increased risk of gastric cancer [39]. SNP in the E-cadherin gene promoter alters transcriptional activity [40] that is associated with scirrhous gastric cancer. The relationship between SNPs and chemosensitivity has also been noted. With progress in the analysis of SNP, the association of specific pathological conditions or sensitivities with various SNPs will be clarified, and, in the near future, information that will lead to the pharmacotherapy and prophylaxis of cancer may be obtained from histopathological samples.
The examination of morphological changes in relation to genetic and epigenetic abnormalities is the main benefit of the genetic analysis of histopathological specimens, and this will improve the quality of histopathological diagnosis. "Histopathology" has discovered minute histological abnormalities, and has closely classified pathological conditions by accumulating knowledge obtained from morphological observations. Another important mission for genetic diagnosis in the field of pathology is to clarify how abnormalities in the functions of the genes and molecules are reflected in morphology. The ultimate objective of molecular pathology is that the morphological abnormalities in all diseases described in the pathology literature will be found to correspond to genetic and epigenetic alterations. In the field of carcinogenesis, molecular pathology must evolve into morphological genomics.

Acknowledgments This work was supported, in part, by Grants-in-Aid for Cancer Research from the Ministry of Education, Culture, Sports, Science, and Technology of Japan; and from the Ministry of Health, Labor, and Welfare of Japan. We would like to thank Dr. S. Usui and Dr. T. Yamagata (Hiroshima City Medical Association) for their continuous support on this project. Special thanks are given to Mr. M. Matsumoto, Mr. T. Kabuto, and other staff members of the Pathology and Cytology Division, Hiroshima City Medical Association Clinical Laboratory, for their skillful technical assistance.

\section{References}

1. Tahara E. Molecular mechanism of stomach carcinogenesis. $\mathbf{J}$ Cancer Res Clin Oncol 1993;119:265-72.

2. Yasui W, Yokozaki H, Fujimoto J, Naka K, Kuniyasu H, Tahara E. Genetic and epigenetic alterations in multistep carcinogenesis of the stomach. J Gastroenterol 2000;35:111-5.

3. Yokozaki H, Yasui W, Tahara E. Genetic and epigenetic changes in stomach cancer. Int Rev Cytol 2001;204:49-95.

4. Yasui W, Ito H, Tahara E. DNA analysis of archival material and its application to tumour pathology. In: Herrington CS, McGee JO'D, editors. Diagnostic molecular pathology, a practical approach. Oxford: Oxford University Press; 1992. p. 193-206.

5. Yokozaki H, Tahara E. PCR analysis of RNA. In: Levy ER, Herrington CS, editors. Non-isotopic methods in molecular biology, a practical approach. Oxford: Oxford University Press; 1995. p. 201-12.

6. Yasui W, Yokozaki H, Tahara E. Molecular diagnosis of gastrointestinal cancer. In: Tahara E, editor. Molecular pathology of gastroenterological cancer: application to clinical practice. Heidelberg Berlin New York Tokyo: Springer; 1997. p. 187-207.

7. Yasui W, Yokozaki H, Shimamoto F, Tahara H, Tahara E. Molecular-pathological diagnosis of gastrointestinal tissues and its contribution to cancer histopathology. Pathol Int 1999;49:76374.

8. Yokozaki H, Shitara Y, Fujimoto J, Hiyama T, Yasui W, Tahara E. Alterations of $p 73$ preferentially occur in gastric adenocarcinomas with foveolar epithelial phenotype. Int J Cancer 1999;83:1926. 
9. Yasui W, Tahara H, Tahara Ej, Fujimoto J, Nakayama J, Ishikawa $\mathrm{F}$, et al. Expression of telomerase catalytic component, telomerase reverse transcriptase, in human gastric carcinomas. Jpn J Cancer Res 1998;89:1099-103.

10. Kim NW, Piatyszek MA, Prowse KR, Harley CB, West MD, Ho $\mathrm{PL}$, et al. Specific association of human telomerase activity with immortal cells and cancer. Science 1994;266:2011-5.

11. Yasui W, Tahara Ej, Tahara H, Fujimoto J, Naka K, Nakayama J, et al. Immunohistochemical detection of human telomerase reverse transcriptase in normal mucosa and precancerous lesions of the stomach. Jpn J Cancer Res 1999;90:589-95.

12. Thibodeau SN, French AJ, Cunningham JM, Tester D, Burgart LJ, Roche PC, et al. Microsatellite instability in colorectal cancer: different mutator phenotypes and the principal involvement of hMLH1. Cancer Res 1998;58:1713-8.

13. Hamamoto T, Yokozaki H, Senba S, Yasui W, Yunotani S, Miyazaki K, et al. Altered microsatellites in incomplete-type intestinal metaplasia adjacent to primary gastric cancers. J Clin Pathol 1997;50:841-6.

14. Nakahara M, Yokozaki H, Yasui W, Dohi K, Tahara E. Identification of concurrent germ-line mutations in $h M S H 2$ and/ or $h M L H 1$ genes in Japanese hereditary non-polyposis colorectal cancer kindreds. Cancer Epidemiol Biomarkers Prev 1997;6: 1057-64.

15. Toyota M, Ahuja N, Suzuki H, Itoh F, Ohe-Toyota M, Imai K, et al. Aberrant methylation in gastric cancer associated with the CpG island methylator phenotype. Cancer Res 1999:59:5438-42.

16. Fleisher AS, Esteller M, Wang S, Tamura G, Suzuki H, Yin J, et al. Hypermethylation of the $h M L H 1$ gene promoter in human gastric cancers with microsatellite instability. Cancer Res 1999; 59:1090-5.

17. Horii A, Han H-J, Shimada M, Yanagisawa A, Kato Y, Ohta H, et al. Frequent replication errors at microsatellite loci in tumors of patients with multiple primary cancers. Cancer Res 1994;54: 3373-5.

18. Yasui W, Kudo Y, Senba S, Yokozaki H, Tahara E. Reduced expression of cyclin-dependent kinase inhibitor p2 $7^{\mathrm{Kip} 1}$ is associated with advanced stage and invasiveness of gastric carcinomas. Jpn J Cancer Res 1997;88:625-9.

19. Suzuki T, Yasui W, Yokozaki H, Naka K, Ishikawa T, Tahara E. Expression of the E2F family in human gastrointestinal carcinomas. Int J Cancer 1999;81:535-8.

20. Merlo A, Herman JG, Lee DJ, Gabrielson E, Burger PC, Baylin $\mathrm{SB}$, et al. 5' $\mathrm{CpG}$ island methylation is associated with transcriptional silencing of the tumor suppressor $p 16 / C D K N 2 / M T S 1$ in human cancers. Nature Med 1995;1:686-92.

21. Grustein M. Histone acetylation in chromatin structure and transcription. Nature 1997;389:349-52.

22. Luo RX, Postigo AA, Dean DC. Rb interacts with histone deacetylase to repress transcription. Cell 1998;92:463-73.

23. Nan $\mathrm{X}, \mathrm{Ng} \mathrm{H}-\mathrm{H}$, Johnson CA, Laherty CD, Turner BM, Eisenman RN, et al. Transcriptional repression by the methylCpG-binding protein $\mathrm{MeCP} 2$ involves a histone deacetylase complex. Nature 1998;393:386-9.

24. Kanai Y, Ushijima S, Kondo Y, Nakanishi Y, Hirohashi S. DNA methyltransferase expression and DNA methylation of CPG islands and peri-centromeric satellite regions in human colorectal and stomach cancers. Int J Cancer 2001;91:205-12.

25. Oue N, Shigeishi H, Kuniyasu H, Yokozaki H, Kuraoka K, Ito R, et al. Promoter hypermethylation of $M G M T$ is associated with protein loss in gastric carcinoma. Int J Cancer 2001;93:805-9.
26. Oue N, Kuraoka K, Kuniyasu H, Yokozaki H, Wakikawa A, Matsusaki K, et al. DNA methylation status of $h M L H 1$, p16INK4a, and $C D H 1$ is not associated with mRNA expression levels of DNA methyltransferase and DNA demethylase in gastric carcinomas. Oncol Rep 2001;8:1085-9.

27. Suzuki T, Kuniyasu H, Hayashi K, Naka K, Yokozaki H, Ono S, et al. Trichostatin A inhibits cell growth and modulates expression of cell cycle- and apoptosis-related molecules in human gastric and oral cancer cell lines. Int J Cancer 2000;88:9927.

28. Fend F, Emmert-Buck MR, Chuaqui R, Cole K, Lee J, Liotta LA, et al. Immuno-LCM: laser capture microdissection of immunostained frozen sections for mRNA analysis. Am J Pathol 1999; 154:61-6.

29. Ochiai A, Hirohashi S. Genetic alterations in the precursors of gastric cancer. In: Tahara E, editor. Molecular pathology of gastroenterological cancer: application to clinical practice. Heidelberg Berlin New York Tokyo: Springer; 1997. p. 43-53.

30. Mori M, Mimori K, Inoue H, Barnard GF, Tsuji K, Nanbara S, et al. Detection of cancer micrometastasis in lymph nodes by reverse transcriptase-polymerase chain reaction. Cancer Res 1995;55: 3417-20.

31. Noguchi S, Hiratsuka M, Furukawa H, Aihara T, Kasugai T, Tamura S, et al. Detection of gastric cancer micrometastases in lymph nodes by amplification of keratin 19 mRNA with reverse transcriptase-polymerase chain reaction. Jpn J Cancer Res 1996; 87:650-4.

32. Nakajo A, Natsugoe S, Ishigami S, Matsumoto M, Nakashima S, Hokita S, et al. Detection and prediction of micrometastasis in the lymph nodes of patients with pNO gastric cancer. Ann Surg Oncol 2001;8:158-62.

33. Nakanishi H, Kodera Y, Yamamura Y, Ito S, Kato T, Ezaki T, et al. Rapid quantitative detection of carcinoembryonic antigenexpressing free tumor cells in the peritoneal cavity of gastriccancer patients with real-time RT-PCR on the LightCycler. Int J Cancer 2000;89:411-7.

34. Mori M, Mimori K, Ueo H, Tsuji K, Shiraishi T, Barnard GF, et al. Clinical significance of molecular detection of carcinoma cells in lymph nodes and peripheral blood by reverse transcriptasepolymerase chain reaction in patients with gastrointestinal or breast carcinomas. J Clin Oncol 1998;16:128-32.

35. International Human Genome Sequencing Consortium. Initial sequencing and analysis of the human genome. Nature 2001;409: 860-921.

36. Venter JC, Adams MD, Myers EW, Richard JM, Sutton GG, Hamilton OS, et al. The sequence of the human genome. Science 2001;291:1304-51

37. The International SNP Map Working Group. A map of human genome sequence variation containing 1.42 million single nucleotide polymorphisms. Nature 2001;409:928-41.

38. Levy-Lahad E, Lahad A, Eisenberg S, Dagan E, Paperna T, Lasinetz $\mathrm{L}$, et al. A single nucleotide polymorphism in the $R A D 51$ gene modifies cancer risk in BRCA2 but not BRCA2 carriers. Proc Natl Acad Sci USA 2001;98:3232-6.

39. El-Omar EM, Carrington M, Chow WH, McColl KE, Bream JH, Young HA, et al. Interleukin-1 polymorphisms associated with increased risk of gastric cancer. Nature 2000;404:398-402.

40. Li L-C, Chui RM, Sasaki M, Nakajima K, Perinchery G, Au HC, et al. A single nucleotide polymorphism in the E-cadherin gene promoter alters transcriptional activities. Cancer Res 2000;60: 873-6. 